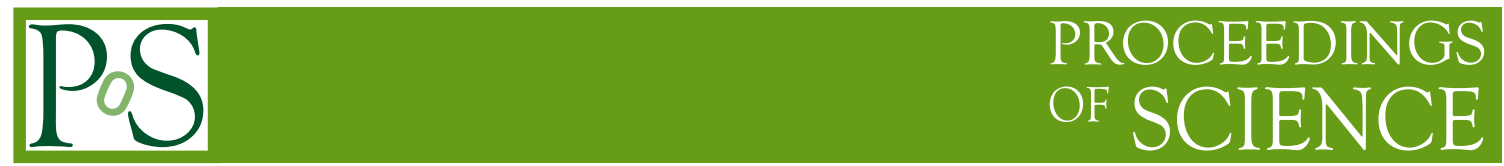

\title{
The $N_{f}=3$ critical endpoint with smeared staggered quarks
}

\author{
Lukas Varnhorst* \\ University of Wuppertal \\ E-mail: L.Varnhorstet-online.de
}

\begin{abstract}
We present preliminary results for the determination of the critical endpoint of the chiral transition in $N_{f}=3$ QCD with $\mu=0$. We have employed unimproved Wilson-Gauge action with staggered fermions, for which previous results on the critical endpoint are available. As an extension of these results we have studied the dependence of the critical mass on the strength of the smearing for small $N_{t}$ and have found a rapid decrease of the critical mass with higher smearing.
\end{abstract}

The 32nd International Symposium on Lattice Field Theory,

23-28 June, 2014

Columbia University New York, NY

${ }^{*}$ Speaker. 


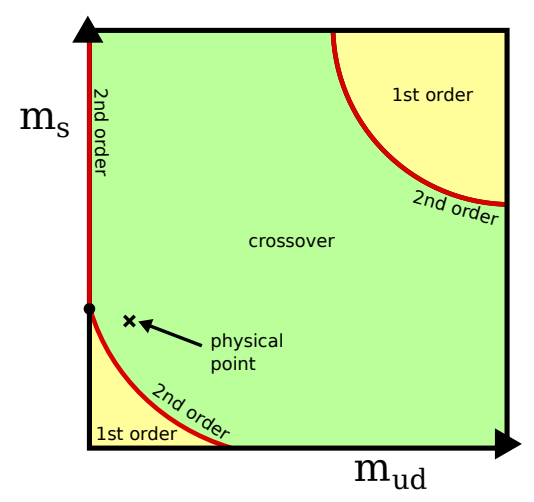

Figure 1: The expected order of the QCD phase transition at $\mu=0$ as a function of the $u, d$ and $s$ quark masses. $[1,2,3]$

\section{Introduction}

It is well known that for the physical values of the quark masses QCD exhibits a analytic crossover at $\mu=0$ from the chiraly broken to the chiraly symmetric phase. However it was predicted [1] that the type of the transition depends on the number of quark flavors and their masses. The expected order of the transition as a function of the quark masses can be found in figure 1 . Here it can be seen that in the case of $N_{f}=3$ degenerate quarks both for infinite and for vanishing quark masses a first order transition is expected. Around this limits regions of first order transition are expected. These regions are separated from the physical region with a crossover behavior by lines of second order transitions. [1,2,3]

There have been several attempts to quantitatively locate at least one point on the line of second order transition in the lower left corner of the $m_{u d} / m_{s}$-quark-mass-plane using lattice QCD. A overview of some of these attempts can be found in table 1. Using different actions and lattice spacings the estimates for the critical masses vary over a large range. In general it seems that for staggered quarks the critical mass shifts to smaller values when the lattice spacing is decreased or the action becomes more continuum like. In recent determination with staggered fermions on $N_{t}=6$ lattices only an upper bound can be given. Recently also results with Wilson fermions are available which predict the critical mass to be somewhat higher than the staggered results [4].

With staggered fermions, a continuum like action and a fine lattice spacing it is very difficult to reach the second order line. On the other hand it is relatively easy to reach this line on coarse lattices with an unimproved action [5, 6]. The approach of this work is to simulate with such an action to locate the transition and then gradually make the action more continuum like. During this procedure the critical masses can be tracked.

More precisely an unimproved Wilson gauge action with 3 degenerate flavors of two times stout-smeared rooted staggered quarks has been used. With the smearing-parameter $\rho=0$ this action coincides with the one used in $[5,6]$. Gradually increasing $\rho$ the behavior of the critical mass has been monitored. 
Table 1: Recent determination of the critical $N_{f}=3$ quark mass with different actions. (Table from [10], with modifications)

\begin{tabular}{llll}
$N_{t}$ & action & $m_{\pi, c}$ & Ref. \\
\hline 4 & stagg.,unimproved & $\sim 260 \mathrm{MeV}$ & {$[5]$} \\
6 & stagg.,unimproved & $\sim 150 \mathrm{MeV}$ & {$[6]$} \\
4 & stagg.,p4 & $\sim 70 \mathrm{MeV}$ & {$[7]$} \\
6 & stagg.,stout & $\leq 50 \mathrm{MeV}$ & {$[8]$} \\
6 & stagg.,HISQ & $\leq 45 \mathrm{MeV}$ & {$[9]$} \\
6 & Wilson-Clover & $\sim 135 \mathrm{MeV}$ & {$[4]$}
\end{tabular}

\section{Methods}

Close to a second order transition observables show universal behavior typical for the universality class of the transition. The transition in question is expected to fall into the $3 \mathrm{D} Z(2)$ universality class, which is the universality class of the 3D ising model. Observables in QCD that show a pronounced transition should, close to the transition, be mixtures of some energy-like observable $\mathscr{E}$ and some magnetisation-like observable $\mathscr{M}$. Thus we can make the ansatz

$$
\begin{gathered}
\mathscr{E}=S_{G} / \beta+a \bar{\psi} \psi \\
\mathscr{M}=\bar{\psi} \psi+b S_{G} / \beta
\end{gathered}
$$

close to the transition. Here $\bar{\psi} \psi$ is the chiral condensate and $S_{G}$ the gluonic action. Using the condition $\operatorname{cov}(\mathscr{E}, \mathscr{M})=0$ the values of $\mathscr{M}$ and $\mathscr{E}$ can be extracted [5]. The histogram of $\mathscr{M}$ and $\mathscr{E}$ can be seen for one simulation very close to the transition in figure 2.

From the behavior of the singular part of the free energy density close to a second order transition in a finite box with volume $L^{3}$ under rescaling one can derive the scaling behavior of the magnetic susceptibility

$$
\chi_{\mathscr{M}}=L^{-\frac{\gamma}{v}} \phi_{\chi \mathscr{M}}^{\mathrm{fss}}\left(c a\left(m-m_{c}\right) L^{\frac{1}{v}}\right) .
$$

$\gamma, v$ are critical exponents and $\phi_{\chi_{M}}^{\mathrm{fss}}$ is the universal finite size scaling function. $a$ is the lattice spacing and $c$ is an undetermined constant. The chiral susceptibility $\chi_{\bar{\psi} \psi}$ can easily be shown to have the same asymptotic scaling behavior than the magnetic susceptibility since $\frac{\gamma}{v}$ is the biggest exponent contributing.

An other quantity to study the phase transition is the binder cummulant. The binder cummulant of an observable $x$ is defined as

$$
B_{4}(x)=\frac{\left\langle x^{4}\right\rangle}{\left\langle x^{2}\right\rangle^{2}}
$$

and is closely connected to the kurtosis. At a critical point in the infinite volume limit the binder cummulant should take a universal value $B_{4}^{*}$ which is characteristic for the universality class of the transition. For larger values of the quark masses it should take the value 3 and for smaller quark masses it should take the value 1 . In the finite volume the jump of $B_{4}$ is washed out. However the 


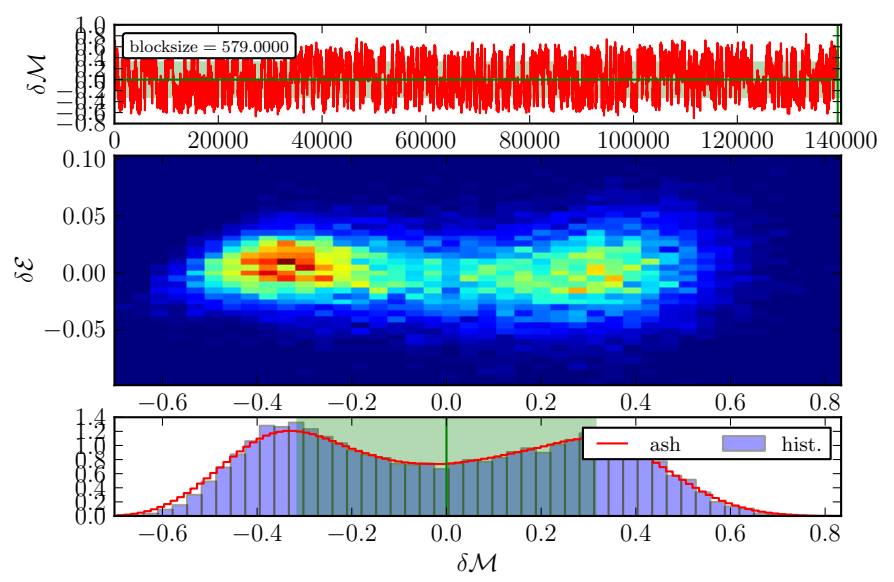

Figure 2: From top to bottom: The time series of the magnetisation like observable $\mathscr{M}$, the two dimensional histogram of $\mathscr{M}$ and $\mathscr{E}$ and the histogram of $\mathscr{M}$. In the histogram the red line is a average shifted histogram.

binder cummulant of $\mathscr{M}$ is expected to scale as

$$
B_{4}(\mathscr{M})=\phi_{B_{4}(\mathscr{M})}^{\mathrm{fss}}\left(c a\left(m-m_{c}\right) L^{\frac{1}{v}}\right) .
$$

From this follows that the for large volumes the value at the crossing point of the binder cummulant calculated at different volumes is an universal quantity and coincides with $B_{4}^{*}$.

The strategy to determine the critical mass which is employed in this work is the following: One generates a set of ensembles for different bare mass parameters. The gauge coupling $\beta$ is tuned for each simulation in such a way that the chiral susceptibility becomes nearly maximal. Using reweighing in $\beta$ one can tune exactly to the maximum of the chiral susceptibility. The line in the $\beta / m a$-plane defined by this procedure is called pseudocritical line.

Along the pseudocritical line susceptibilities should behave according to equation (2.2). A particularly easy way to determine the critical endpoint is to rescale the susceptibility by a factor $L^{\frac{\gamma}{v}}$ and to look for an intersection point. Doing so one has to make an ansatz for the $\phi_{\chi \mathscr{M}}^{\mathrm{fss}}$. In this exploratory work a Pade-ansatz

$$
\phi_{\chi \mathscr{H}}^{\mathrm{fss}}=\frac{1}{\alpha+x} \quad \text { with } \quad x=a\left(m-m_{c}\right)
$$

where $\alpha$ is a free fit parameter was used. A exponential ansatz leads to comparable results. With the exponents are $\gamma$ and $v$ fixed to their $Z(2)$ values one is left only with the constant $c$ from equation (2.2), the parameter $\alpha$ and the critical mass which have to be fitted. Analyses for several values of the stout-smearing parameter has been combined into a joint fit in which it was demanded that the scaling function from equation (2.5) is the same for all values of $\rho$.

For the binder cummulant scaling function $\phi_{B_{4}(\mathscr{M})}^{\mathrm{fss}}$ a polynomial ansatz of degree 2 was employed and the critical exponents where kept as free fit parameters. Therefore the universality class in principle could be determined from the values of $B_{4}^{*}$. It turned out that the fits with the limited dataset and unconstrained critical exponents are relatively unstable. Therefore and because of limited space only the critical masses as determined form the scaling of the susceptibility are presented. 


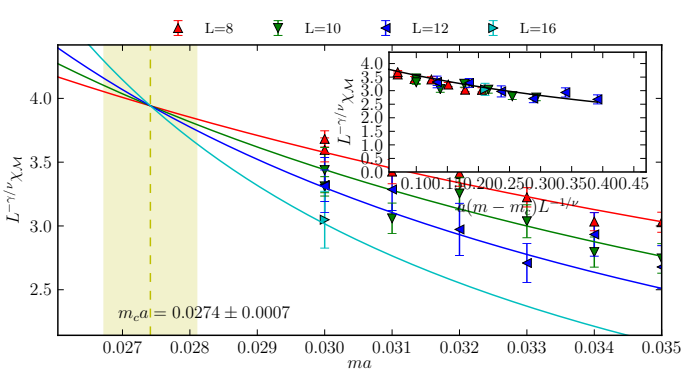

(a) $\rho=0.00$

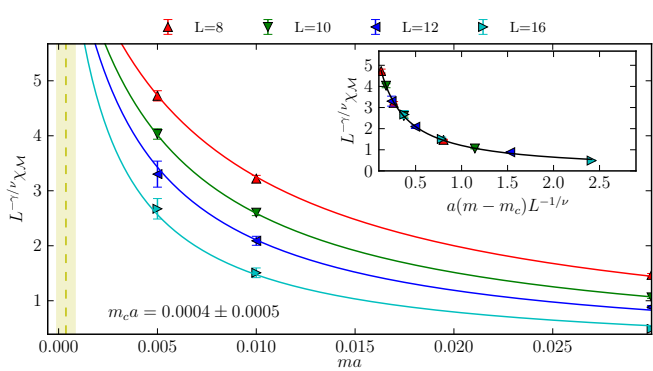

(c) $\rho=0.04$

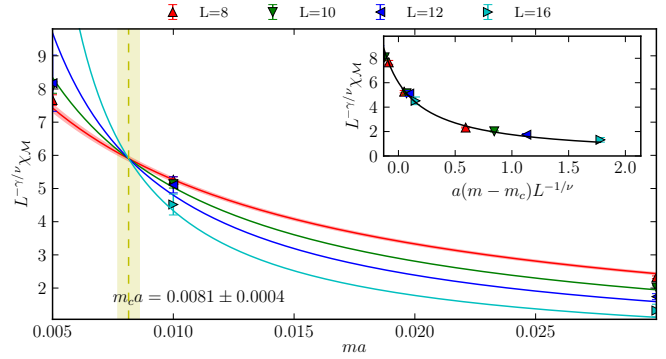

(b) $\rho=0.02$

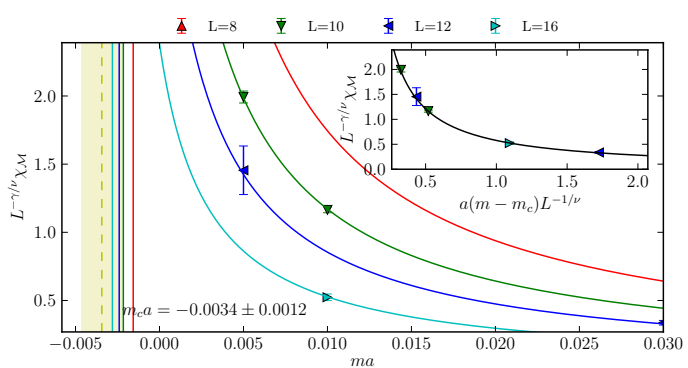

(d) $\rho=0.08$

Figure 3: The scaling of $\chi_{\mathscr{M}}$ at four different smearing parameters at $N_{t}=4$. The $y$-axis has been rescaled with proper critical exponents. The different curves correspond to different volumes. The yellow band indicates the estimate for the critical mass. The inset shows the universal scaling function with the data collapsed to the curves by proper rescaling of the $x$ - and $y$-axis.

\section{Results}

Simulations where performed at four values of the smearing parameter $\rho$ at $N_{t}=4$. For each $\rho$ three bare quark masses and four spatial lattice extends $L$ where chosen. In figure 3 the scaling of $\chi_{\mathscr{M}}$ is plotted.

In figure 4 the estimate for the critical mass at different values of the smearing parameter is shown. One can see that on $N_{t}=4$ lattices the critical mass decreases significantly already for very small values of the smearing parameter. Eventually even negative values are encountered. The meaning of these negative values are as follows: There is no positive mass at which the transition is present. However remnants of the $Z(2)$-scaling can still be existent. When fitted with the same fit function, the critical mass will shift to negative values.

A similar analysis was also performed with $N_{t}=6$ data. Since the cost of the simulations increases drastically form $N_{t}=4$ to $N_{t}=6$ there exist only a limited amount of statistics and only two values of the smearing parameter and the spatial lattice extend. Because of this the results for $N_{t}=6$ lattices might not be very reliable and where only included for illustrative purpose. The results of the fit to $\chi_{\mathscr{M}}$ can be seen if figure 5. The data from $N_{t}=6$ suggest a much milder shift of the critical mass as function of the smearing parameter than observed at $N_{t}=4$ lattices.

In figure 6 several scenarios for the continuum extrapolation of the critical mass are shown. Scenario (a) is the one commonly expected. However the scenarios (b)-(d) are also possible and 


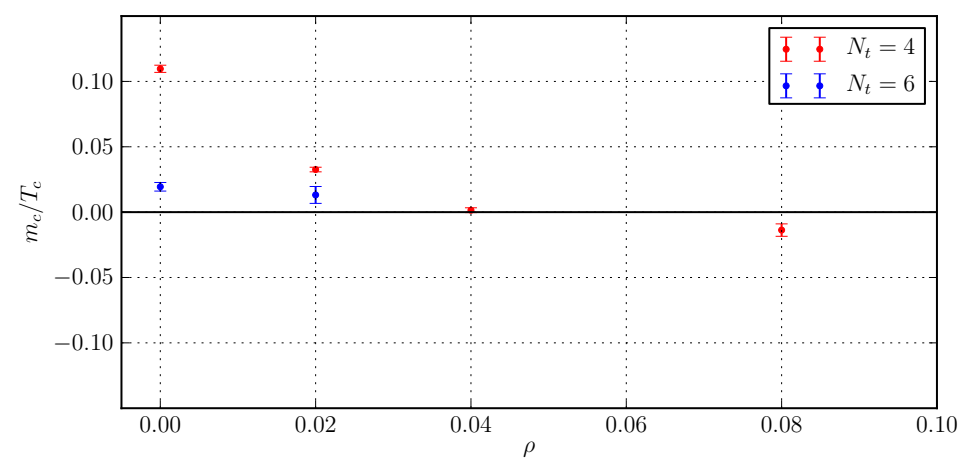

Figure 4: Estimates of the critical mass as the function of the smearing parameter.

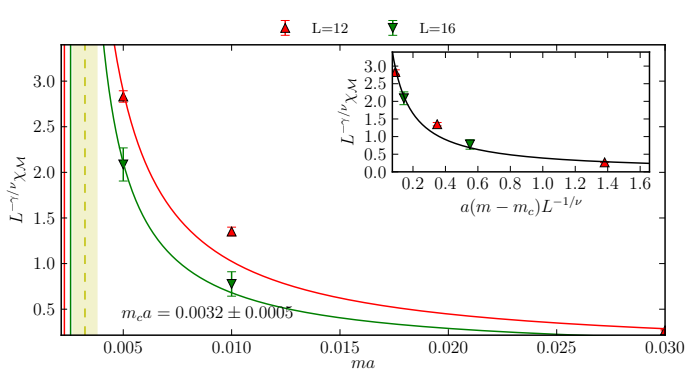

(a) $\rho=0.00$

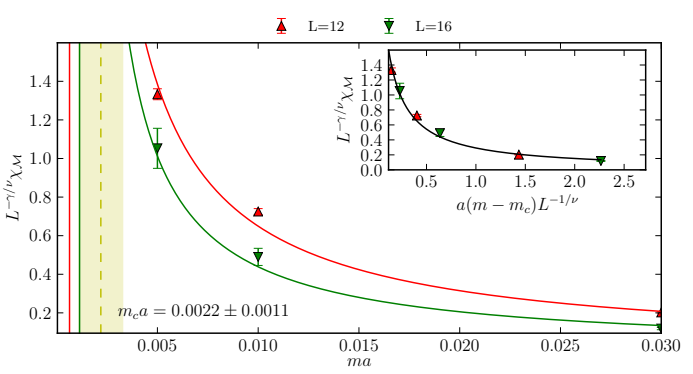

(b) $\rho=0.02$

Figure 5: The scaling of $\chi_{\mathscr{M}}$ at two different smearing parameters at $N_{t}=6$. The $y$-axis has been rescaled with proper critical exponents. The different curves correspond to different volumes. The yellow band indicates the estimate for the critical mass. The inset shows the universal scaling function with the data collapsed to the curves by proper rescaling of the $x$ - and $y$-axis.

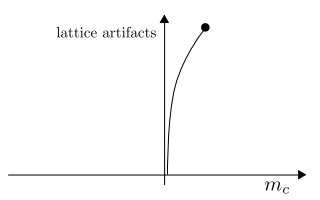

(a)

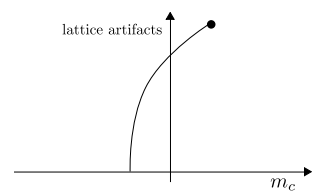

(b)

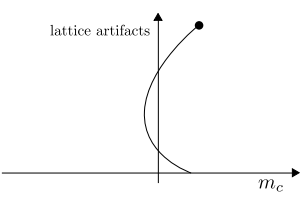

(c)

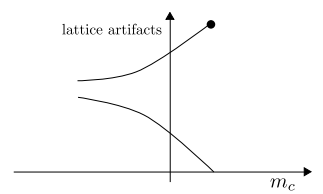

(d)

Figure 6: Several scenarios for the continuum extrapolation of the critical mass value.

will result in the transition to be unobservable at intermediate lattice spacings. This is what seems to happen with a large smearing parameter.

The results presented above might be subject to some systematic uncertainties: The volumes are relatively small, so that finite volume effects might influence the analysis. Therefore in the future more and bigger volumes should be used. This is especially true for small masses. Also it might be beneficial to introduce more values of the quark mass. This is expected to stabilize the fits to the binder cummulant which could than be used as a check whether the transition found is in the $Z(2)$ universality class. Finally one might use a refined ansatz for the fit function. 
Although the continuum value for the critical mass must be independent from the smearing parameter it might be interesting to investigate whether one can find a relation between the smearing dependence of the critical mass at several lattice spacings. If one is able to find a value of $\rho$ at which the critical mass decreases towards the continuum and one at which it increases it might be possible to control the continuum limit from two sides. An other strategy might be to perform simulations at a value of $\rho$ at which the $N_{t}=4$ - and $N_{t}=6$-value are the same to get a flatter continuum extrapolation. Also it would be interesting to investigate whether similar observation can be made for different actions.

\section{Summary}

The behavior of the critical mass of the second order endpoint at low quark mass and $\mu=0$ in the $N_{f}=3$ case for an unimproved Wilson gauge action and stout smeared staggered quarks was studied. It was found that it shows a pronounced dependence on the smearing parameter $\rho$ at $N_{t}=4$ lattices and a much milder behavior at $N_{t}=6$ lattices. There are indications that the transition vanishes for certain smearing parameters on $N_{t}=4$ lattices. To confirm the presented results further investigations using more volumes and/or quark masses are required

\section{Acknowledgement}

The author would like to thank Zoltán Fodor and Christian Hölbling for the stimulating suggestions and continuous support throughout the project. This work is supported in part by the DFG grant SFB/TR55.

\section{References}

[1] R. D. Pisarski and F. Wilczek, Phys. Rev. D 29 (1984) 338.

[2] F. R. Brown, F. P. Butler, H. Chen, N. H. Christ, Z. -h. Dong, W. Schaffer, L. I. Unger and A. Vaccarino, Phys. Rev. Lett. 65 (1990) 2491

[3] Y. Aoki, G. Endrodi, Z. Fodor, S. D. Katz and K. K. Szabo, Nature 443 (2006) 675

[4] X. Y. Jin, Y. Kuramashi, Y. Nakamura, S. Takeda and A. Ukawa, arXiv:1411.7461 [hep-lat].

[5] Karsch, Laermann and Schmidt, Phys. Lett. B 520 (2001) 41

[6] de Forcrand, Kim and Philipsen, PoS LAT 2007 (2007) 178

[7] F. Karsch, C. R. Allton, S. Ejiri, S. J. Hands, O. Kaczmarek, E. Laermann and C. Schmidt, Nucl. Phys. Proc. Suppl. 129 (2004) 614

[8] G. Endrodi, Z. Fodor, S. D. Katz and K. K. Szabo, PoS LAT 2007 (2007) 182

[9] H. -T. Ding, A. Bazavov, P. Hegde, F. Karsch, S. Mukherjee and P. Petreczky, PoS LATTICE 2011 (2011) 191

[10] K. Szabo, PoS LAT 2014014 